\title{
The roles of ARHGAP10 in the proliferation, migration and invasion of lung cancer cells
}

\author{
JI-PING TENG ${ }^{1,2}$, ZHI-YING YANG ${ }^{2}$, YU-MING ZHU ${ }^{3}$, DA NI $^{2}$, ZHI-JUN ZHU ${ }^{2}$ and XIAO-QIANG LI ${ }^{1}$ \\ ${ }^{1}$ Department of Vascular Surgery, The Second Affiliated Hospital of Soochow University, Suzhou, \\ Jiangsu 215004; ${ }^{2}$ Department of Thoracic and Cardiovascular Surgery, Shanghai Ninth People's Hospital, \\ Shanghai Jiao Tong University School of Medicine, Shanghai 200011; ${ }^{3}$ Department of \\ Thoracic Surgery, Shanghai Pulmonary Hospital, Shanghai 200433, P.R. China
}

Received May 22, 2017; Accepted August 1, 2017

DOI: $10.3892 / \mathrm{ol} .2017 .6729$

\begin{abstract}
Lung cancer is a leading cause of cancer-related mortalities worldwide. In the present study, a comparison of To determine the roles of ARHGAP10 in the proliferation, migration and invasion of lung cancer cells expression levels between normal lung tissues and lung cancer tissues were compared using immunoblotting, and CCK-8 and Transwell assays. Lung cancer tissues had a decreased ARHGAP10 mRNA expression level compared to the adjacent normal tissues. The ectopic expression of ARHGAP10 significantly suppressed the migration, invasion and proliferation of lung cancer cells. Gene set enrichment analysis revealed that metastasis and Wnt signaling pathways were negatively correlated with ARHGAP10 expression. Immunoblotting analysis revealed that ARHGAP10 overexpression inhibited metastasis [matrix metalloproteinase (MMP)-2, MMP-9 and VEGF] and the expression of Wnt pathway-related proteins $(\beta$-catenin and $\mathrm{c}-\mathrm{Myc})$. Moreover, the stimulation effects of lithium chloride, a GSK3 $\beta$ inhibitor, on the accumulation of $\beta$-catenin were notably suppressed by ARHGAP10 overexpression. Collectively, ARHGAP10 acts to suppress tumor within lung cancer by affecting metastasis and Wnt signaling pathways. The results therefore suggest that ARHGAP10 is a potentially attractive target for the treatment of lung cancer.
\end{abstract}

\section{Introduction}

Lung cancer is a leading cause of cancer-related mortality worldwide. The most frequent type of lung cancer is non-small cell lung cancer (NSCLC) (1). Despite improvements in the therapeutic strategies of lung cancer, long-term survival is far from satisfactory for lung cancer patients mainly due to

Correspondence to: Dr Xiao-Qiang Li, Department of Vascular Surgery, The Second Affiliated Hospital of Soochow University, 1055 Sanxiang Road, Suzhou, Jiangsu 215004, P.R. China

E-mail: xiaoqianglisz1@126.com; xiaoqianglisz@126.com

Key words: ARHGAP10, lung cancer, Wnt, metastasis recurrence and metastasis (2). The molecular mechanisms of lung carcinogenesis have yet to be completely clarified (3-7).

ARHGAP10 belongs to the family of Rho GTPase activating proteins (RhoGAP), which catalyze the hydrolysis of the active Rho GTPases (GTP-bound) to the inactive form (GDP-bound), and thus impedes Rho GTPases-regulated biological procedures, including migration, cytoskeleton organization, cell proliferation and gene transcription (8). It has been reported that ARHGAP10 acts as a RhoGAP for several Rho GTPases, including Cdc42 (9), RhoA and RhoC (10). ARHGAP10 contains a pleckstrin homology (PH) domain and a PSD95/ DglA/ZO-1-like (PDZ) domain. The two domains are essential for protein-protein interaction. A number of proteins, including $\alpha$-catenin (11), ARF1 (9), FAK, PKC- $\zeta$ (12) and $\beta$-arrestin 1 (13), have been identified as ARHGAP10 interaction proteins. By binding with these proteins, ARHGAP10 actively participated in the regulation of the formation of cell junction and stress fiber, vesicular transport of Golgi membranes and influenza virus replication. Previous findings showed that ARHGAP10 is associated with paediatric leukaemia (14), invasive breast cancer (15) and ovarian cancer (16). The present study aimed to investigate the biological functions and expression of ARHGAP10 in lung cancer.

In the present study, we compared ARHGAP10 expression level between normal lung tissues and lung cancer ones. The effects of ARHGAP10 overexpression on cell migration, invasion and proliferation of lung cancer cell lines were investigated. Furthermore, gene set enrichment analysis (GSEA) and immunoblot analyses suggested the metastasis and Wnt signaling pathways were negatively correlated with ARHGAP10 expression. In conclusion, our data indicated that ARHGAP10 may be a tumor suppressor protein for lung cancer.

\section{Materials and methods}

Tissue samples. The study was approved by the Ethics Committee of the Second Affiliated Hospital of Soochow University (Shanghai, China). In total, 35 pathologically confirmed lung cancer patients who had received surgery at the Second Affiliated Hospital of Soochow University between January 2010 and December 2011 were enrolled in the present study after obtaining of their written informed consent. The 
same patients provided lung cancer tissues along with adjacent normal ones. The paired tissues were stored at $-80^{\circ} \mathrm{C}$ after being snap-frozen.

Cell culture. We purchased the wild-type cancer cell lines of human lung (A549, NCI-1975 and NCI-H1299) from the Cell Bank of the Shanghai Institutes of Biological Sciences (Shanghai, China), which were maintained with $5 \% \mathrm{CO}_{2}$ at $37^{\circ} \mathrm{C}$. RPMI-1640 medium (HyClone, Logan, UT, USA) was used to grow the cell lines with supplementation of $1 \%$ antibiotics and $10 \%$ fetal bovine serum (FBS; Gibco, Carlsbad, CA, USA).

RNA extraction, cDNA synthesis and reverse transcription-quantitative PCR. RNA isolated by TRIzol reagent (Invitrogen, Carlsbad, CA, USA) was treated with DNase and then subjected to reverse transcription reaction employing reverse transcriptase M-MLV and oligo(dT) (21-mer) (Takara, Dalian, China). RT-qPCR was conducted to quantify the mRNA level of ARHGAP10 using a SYBR ${ }^{\circledR}$ Green Master kit (Thermo Fisher Scientific, Inc., Waltham, MA, USA) with an ABI 7300 instrument (Applied Biosystems, Foster City, CA, USA) based on the manufacturer's protocols. The PCR program used was: $95^{\circ} \mathrm{C}$ for $10 \mathrm{~min}$, followed by 40 cycles of $95^{\circ} \mathrm{C}$ for $15 \mathrm{sec}$ and $60^{\circ} \mathrm{C}$ for $45 \mathrm{sec}$. The primer sequences used in the present study have been previously described (16). mRNA values of ARHGAP10 were normalized to the internal control GAPDH. Each sample was run in triplicate and confirmed by at least three replicates.

Immunoblotting assay. Total cell lysates isolated using RIPA buffer containing protease inhibiting agent and phosphatase inhibitor (Beyotime, Shanghai, China) were separated by $10 \%$ SDS-PAGE, and then transferred ontonitrocellulose membranes (Millipore, Bredford, MA, USA), which were blocked in skim milk at room temperature for $1 \mathrm{~h}$. The membranes were then probed with primary antibodies against ARHGAP10 (1:150; Santa Cruz Biotechnology, Inc., Santa Cruz, CA, USA), matrix metalloproteinase (MMP)-2 (1:1,000), MMP-9 (1:1,000), VEGF $(1: 1,000), \beta$-catenin $(1: 1,000)$, c-Myc $(1: 1,000), \mathrm{p} 21$ $(1: 1,000)$ (all from Abcam, Cambridge, MA, USA) or GAPDH (1:1,500; Cell Signaling Technology, Inc., Danvers, MA, USA) overnight in gentle shaking at $4^{\circ} \mathrm{C}$. The membranes were then incubated for $1 \mathrm{~h}$ at room temperature with the corresponding HRP-conjugated secondary antibodies (Beyotime). An enhanced chemiluminescence kit (Millipore) was used to visualize signals while ImageJ software quantified the band density, which was normalized to GAPDH.

Cell proliferation analysis. The Cell Counting Kit-8 (CCK-8) assay kit (Beyotime) was used to measure the cell proliferation rate. Cells were cultured in 6-well plates under normal culture conditions. The cells were infected with ARHGAP10 expression virus or control vector virus. After $24 \mathrm{~h}$, the cells were trypsinized and then plated in $96-$-well plates $\left(2 \times 10^{3}\right.$ cells/well). After incubation with CCK-8 solution at room temperature for $1 \mathrm{~h}$ at the indicated time points $(0,24,48$ and $72 \mathrm{~h})$, the optical density (OD) was measured at a wavelength of $450 \mathrm{~nm}$ according to the manufacturer's instructions. Each experiment was repeated three times.
Transwell assay. Cell invasion and migration were evaluated using Transwell assays performed in the Boyden chamber with and without Matrigel coating (BD Biosciences, Franklin Lakes, NJ, USA), respectively. Cells were cultured in 6-well plates, which were then infected with ARHGAP10 expression virus or control vector virus. The cells were trypsinized, suspended in RPMI-1640 medium containing 0.1\% FBS and plated on the upper chambers (1.0x10 ${ }^{5}$ cells) after $24 \mathrm{~h}$. RPMI-1640 medium with $10 \%$ FBS was contained in the lower chambers. The cells adhering to the upper chambers were carefully scrubbed off after incubation for $24 \mathrm{~h}$. After fixation with $10 \%$ formalin and staining with $1 \%$ gentian violet, the cells migrating onto the lower surface were counted under an inverted microscope (AZ100; Nikon, Tokyo, Japan). Each experiment was run in triplicate.

GSEA. We downloaded the dataset of TCGA lung cancer from The Cancer Genome Atlas project (TCGA, https://tcga-data. nci.nih.gov/tcga/) and analyzed this dataset using the software GSEA v2.2.2 (www.broadinstitute.org/gsea), as previously described (17). In the present study, the samples were classified as ARHGAP10 low and ARHGAP10 high to annotate phenotype. The gene sets used to conduct GSEA were obtained from the Molecular Signatures Database (http://www.broad.mit. edu/gsea/msigdb/index.jsp).

Lithium chloride ( $\mathrm{LiCl}$ ) treatment. A549 cells were plated in 6-well plates and infected with ARHGAP10 expression virus or control vector virus. After $24 \mathrm{~h}$, the cells were treated with or without $10 \mathrm{mM} \mathrm{LiCl}$ for $24 \mathrm{~h}$, and then collected for immunoblotting analysis for $\beta$-catenin expression.

Statistical analysis. GraphPad Prism 6.0 software (GraphPad, San Diego, CA, USA) was employed to perform statistical analyses. The paired t-test was used to analyze differences in ARHGAP10 expression of lung cancer tissues from adjacent normal ones. Analysis was conducted by one-way analysis of variance (ANOVA) for in vitro cell experiments. $\mathrm{P}<0.05$ was considered to indicate a statistically significant difference.

\section{Results}

ARHGP10 is downregulated in lung cancer tissues. First, we evaluated ARHGAP10 mRNA levels in lung cancer tissues as well as paired adjacent normal tissues of 35 patients by RT-qPCR. The mRNA levels of ARHGAP10 were more downregulated in tumor tissues than that in adjacent normal tissues (Fig. 1A). Moreover, a publically available dataset from TCGA demonstrated that lung cancer tissues had a lower ARHGAP10 expression level than that of normal tissues, which was consistent with our findings (Fig. 1B).

Ectopic expression of ARHGAP10 in lung cancer cells. We analyzed the ARHGAP10 expression within the four lung cancer cells. The protein and mRNA levels of ARHGAP10 in NCI-H460 and A549 cells were relatively lower (Fig. 2A and B) and selected for further overexpression experiments.

ARHGAP10-expressing lentiviral plasmid was constructed and lentivirus was produced to infect A549 and NCI-H460 cells. ARHGAP10 expression in these cell lines was quantified using RT-qPCR and immunoblotting. As shown 
A

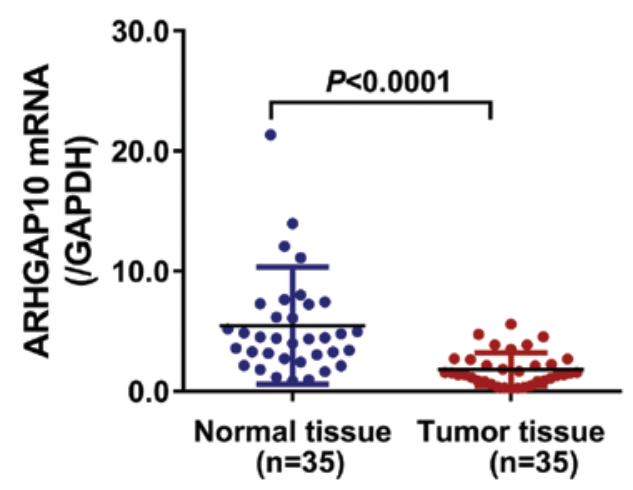

B

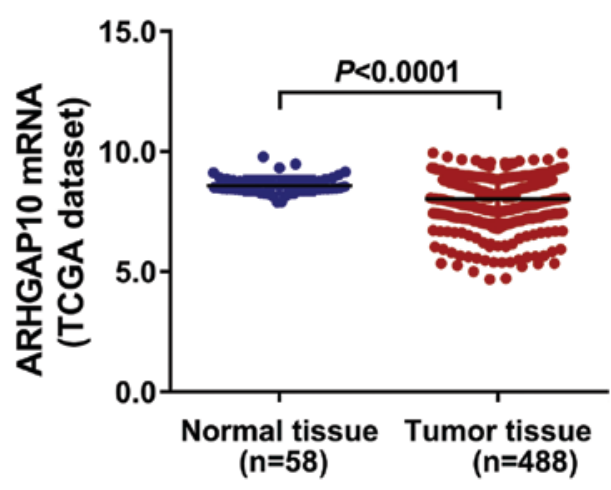

Figure 1. ARHGAP10 expression is downregulated in cancer tissues of human lung. (A) ARHGAP10 mRNA levels of 35 lung cancer tissues and paired normal ones. (B) mRNA levels in ARHGAP10 of normal lung tissues and lung cancer tissues from the TCGA dataset.

A

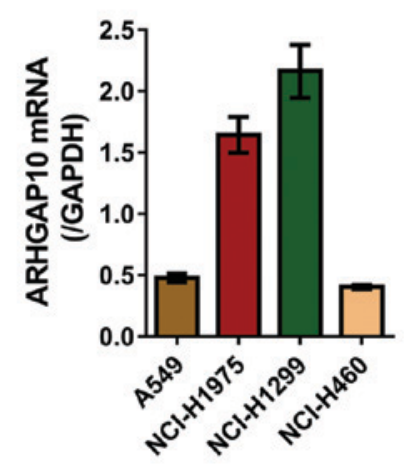

B
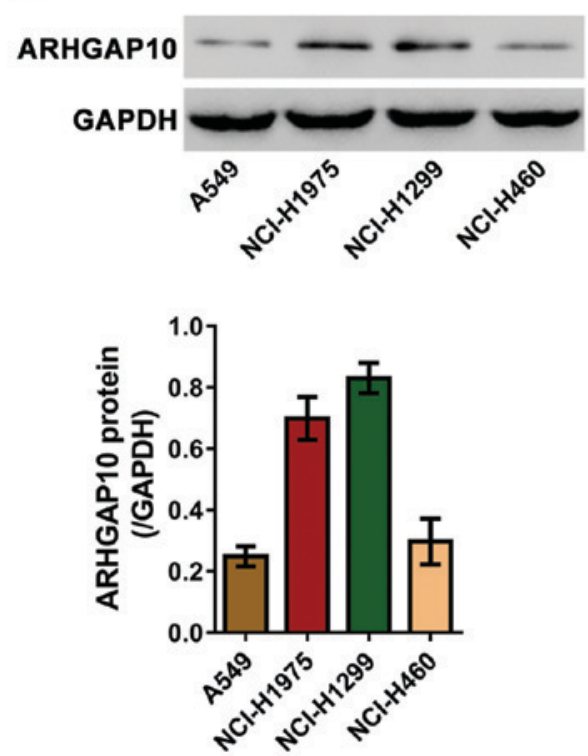

C

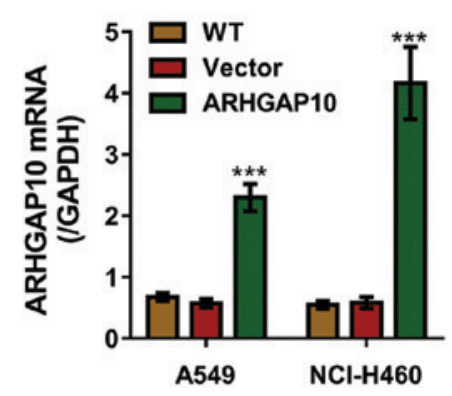

D
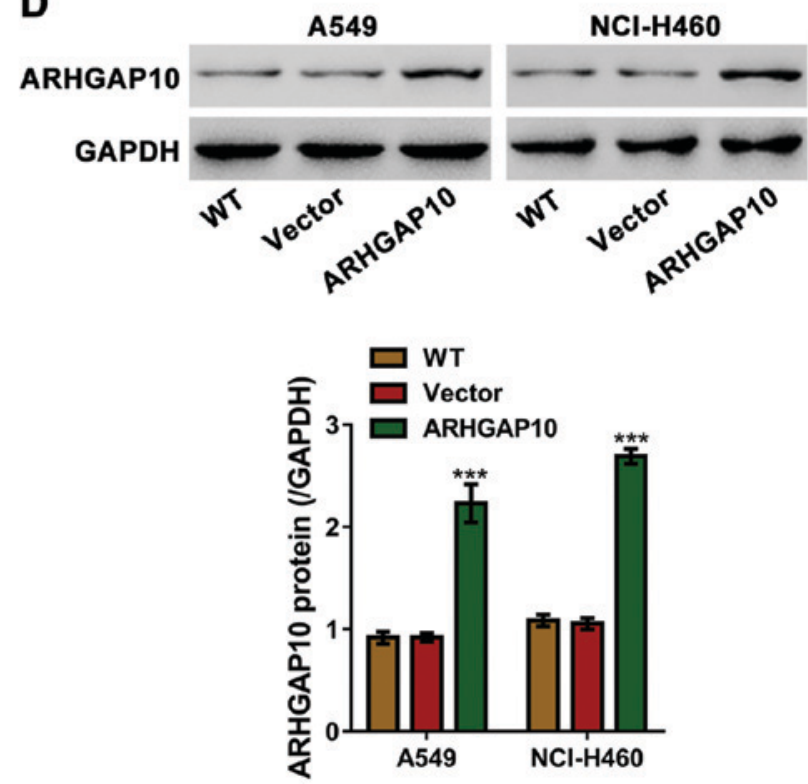

Figure 2. Ectopic expression of ARHGAP10 within lung cancer cell lines. (A and B) RT-qPCR and immunoblot analyses of ARHGAP10 protein and mRNA levels within lung cancer cell lines (A549, NCI-H1975, NCI-H1299 and NCI-H460). GAPDH served as an internal inhibition. (C and D) ARHGAP10 protein and mRNA levels in NCI-H460 and A549 cells infected with ARHGAP10-expressing virus or control vector virus. ${ }^{* * * *} \mathrm{P}<0.001$.

in Fig. 2C and D, control vector lentiviral infection (Vector) had no effects on ARHGAP10 expression as compared to the wild-type (WT) cells. ARHGAP10-expressing lentiviral infection significantly enhanced ARHGAP10 expression of the protein and mRNA levels in contrast to WT cells and cells infected with vector virus.
Ectopic ARHGAP10 expression inhibits lung cancer cell proliferation. CCK-8 assay was employed to measure the effects of ARHGAP10 on cell multiplication. As shown in Fig. 3, the cell multiplication rate of NCI-H460 and A549 cells overexpressing ARHGAP10 was significantly higher than the WT cells and cells infected with vector virus. 
A

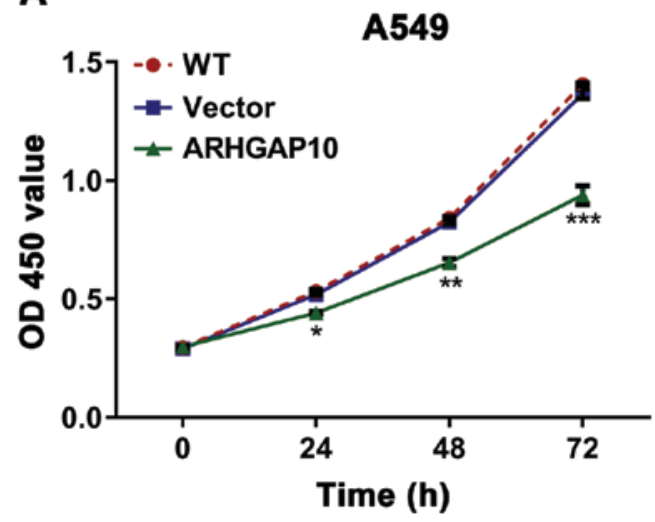

B

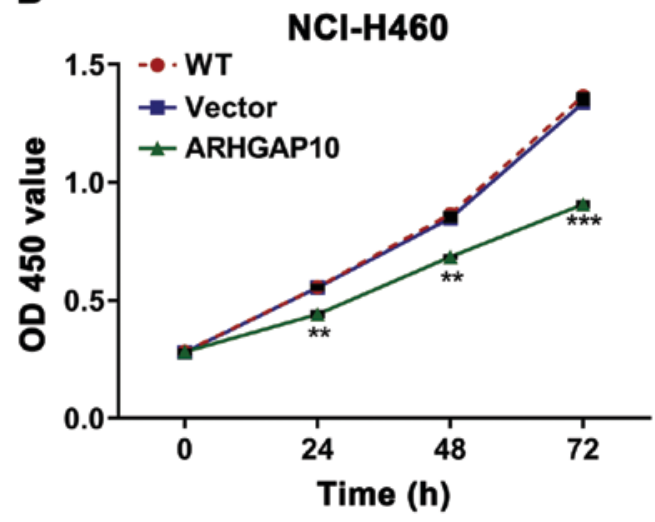

Figure 3. Overexpression of ARHGAP10 inhibits the proliferation of lung cancer cells. CCK-8 assay of cell proliferation in (B) NCI-H460 and (A) A549 cells at 24,48 and $72 \mathrm{~h} .{ }^{*} \mathrm{P}<0.05,{ }^{* * *} \mathrm{P}<0.01,{ }^{* * *} \mathrm{P}<0.001$.

A

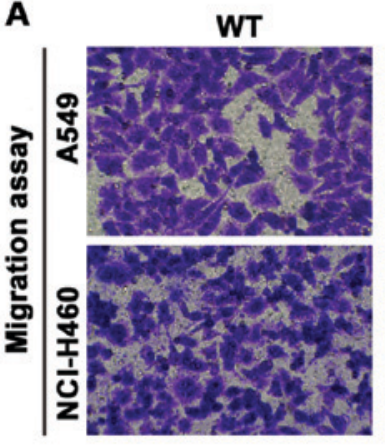

B

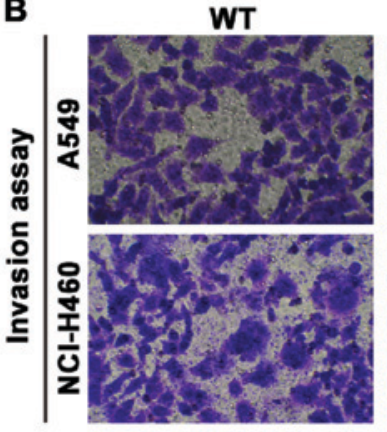

Vector

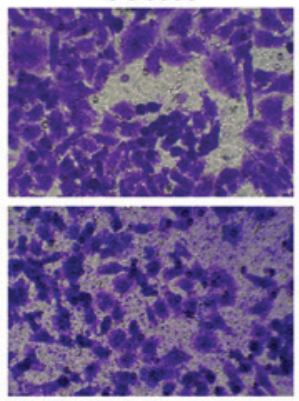

Vector

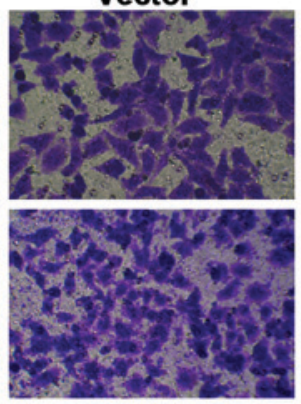

ARHGAP10

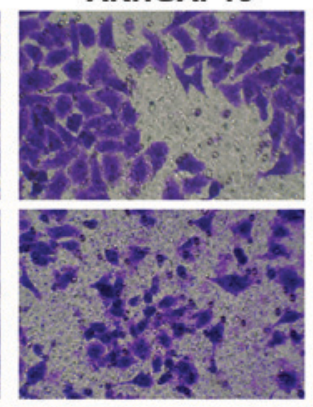

ARHGAP10

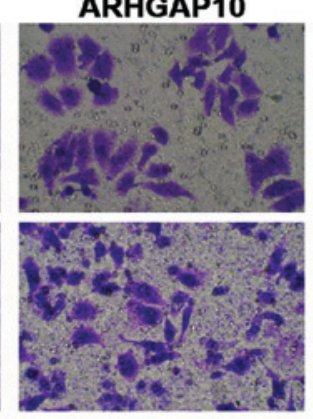

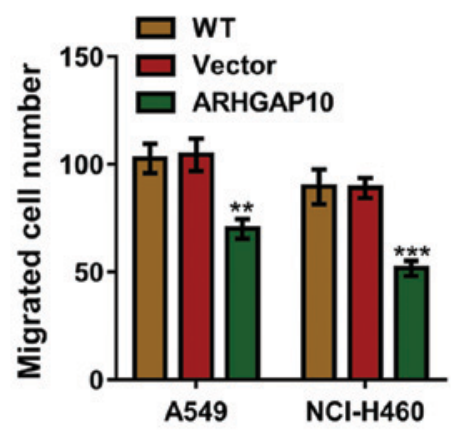

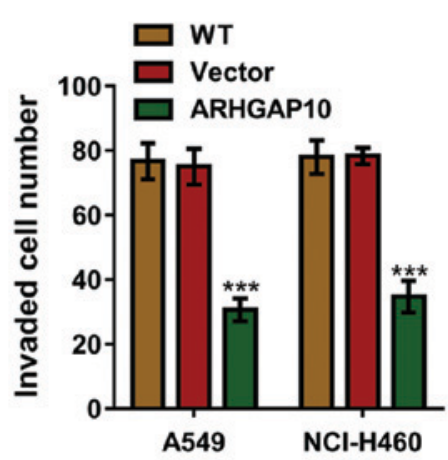

Figure 4. ARHGAP10 inhibits lung cancer cell invasion and migration. Transwell assays were performed in Boyden chamber without (A) or with (B) Matrigel coating to analyze the impacts of ARHGAP10 on the invasion and migration of lung cancer cells. The number of cells migrating onto the lower surfaces is shown. ${ }^{* * *} \mathrm{P}<0.001$, and ${ }^{* *} \mathrm{P}<0.01$.

ARHGAP10 suppresses the invasion and migration of lung cancer cells. The effects that ARHGAP10 produced on the invasion and migration of lung cancer cells were assessed using Transwell assays (Fig. 4). ARHGAP10 overexpression in NCI-H460 and A549 cells significantly reduced the invasion and migration capacity as compared to control cells (WT and vector).

GSEA for ARHGAP10 expression in lung cancer. GSEA was performed to investigate pathways that were associated with the different ARHGAP10 expression levels (Fig. 5A). Metastasis and Wnt signaling pathways were negatively correlated with ARHGAP10 expression.
The effects of ARHGAP10 overexpression on the expression of key proteins of metastasis (MMP-2, MMP-9 and VEGF) and Wnt signaling pathways ( $\beta$-catenin, $\mathrm{c}-\mathrm{Myc}$ and $\mathrm{p} 21)$ were then assessed in A549 and NCI-H460 cells. In contrast to the control cells, the mRNA and proteins levels of MMP-9,MMP-2, $\beta$-catenin as well as c-Myc were significantly increased in lung cancer cells with ARHGAP10 overexpression, while those of p21 were notably decreased (Fig. 5B and C). These data validated the results of GSEA.

$\mathrm{LiCl}$, a GSK3 $\beta$ inhibitor, is able to enhance the activation of Wnt canonical signaling (18). To further explore the effects of ARHGAP10 on Wnt signaling, A549 cells were infected with ARHGAP10-expressing lentivirus or vector lentivirus, 
A

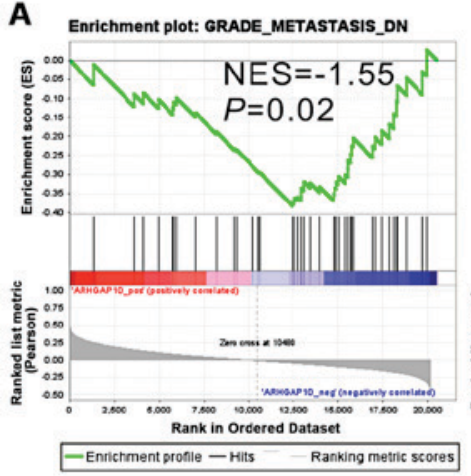

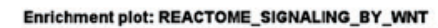

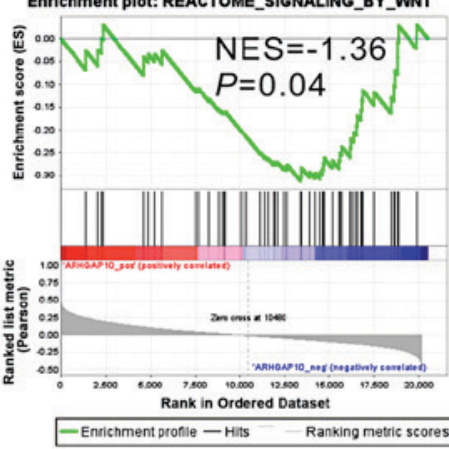

C
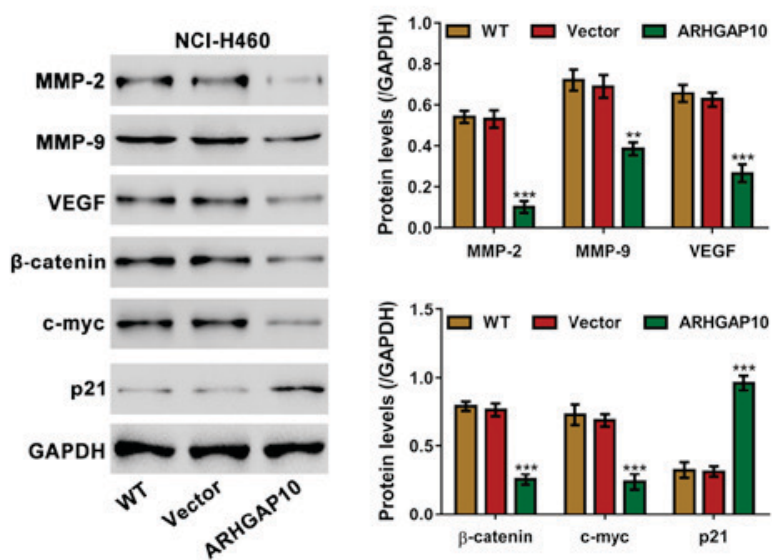

D
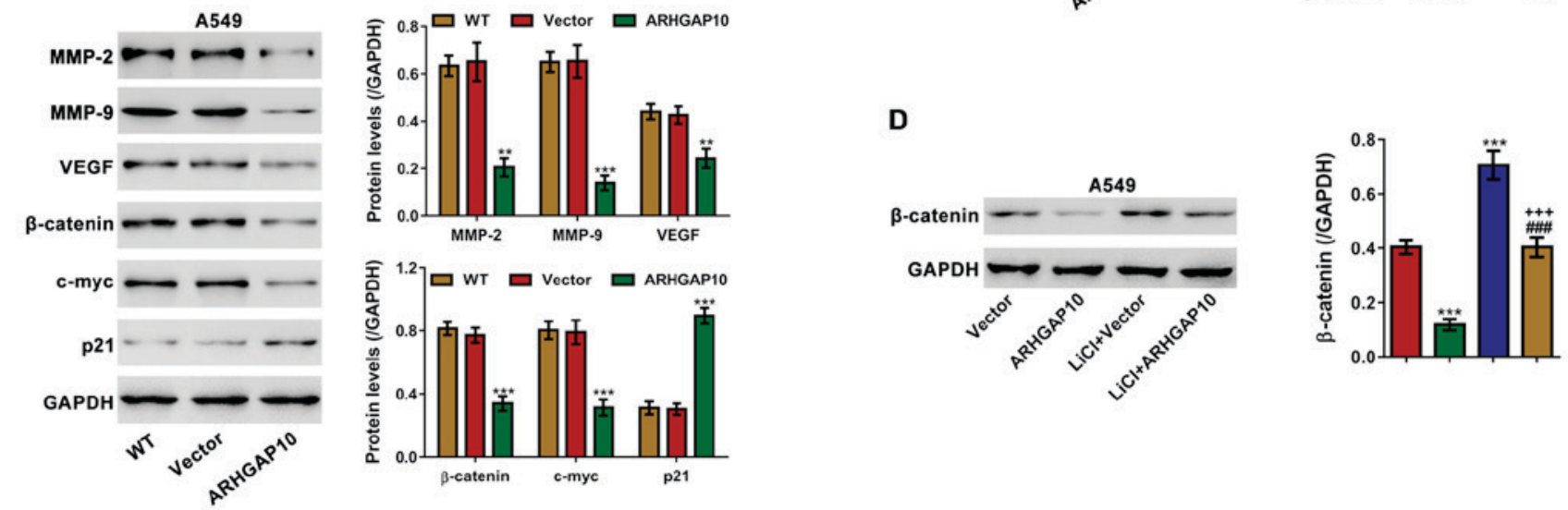

Figure 5. (A) The GSEA results showing the correlation of ARHGAP10 levels and metastasis and Wnt signaling pathways. (B and C) Immunoblot analysis for key regulators of metastasis and Wnt signaling pathways in (B) A549 and (C) NCI-H460 cells infected with ARHGAP10-expressing virus or control vector virus for $48 \mathrm{~h} .{ }^{* *} \mathrm{P}<0.01,{ }^{* * *} \mathrm{P}<0.001$. (D) Immunoblot analysis for $\beta$-catenin expression in A549 cells infected with ARHGAP10-expressing virus or control vector virus and then treated with $10 \mathrm{mM} \mathrm{LiCl} .{ }^{* * *} \mathrm{P}<0.001$ vs. vector; ${ }^{\# \# \#} \mathrm{P}<0.001$ vs. ARHGAP10 overexpression; ${ }^{+++} \mathrm{P}<0.001$ vs. $\mathrm{LiCl}+$ vector. GSEA, gene set enrichment analysis; $\mathrm{LiCl}$, lithium chloride; NES, normalized enrichment score.

and then stimulated with $10 \mathrm{mM} \mathrm{LiCl}$ (Fig. 5D). LiCl stimulation led to the accumulation of $\beta$-catenin, which was significantly rescued by ARHGAP10 overexpression. These results suggested that ARHGAP10 significantly inhibited Wnt/ $\beta$-catenin signaling.

\section{Discussion}

Recent studies have suggested the association between ARHGAP10 and various types of cancer $(10,14-16,19,20)$. For example, Wong et al reported the methylation of ARHGAP10 in paediatric leukaemia (14). Azzato et al performed a genome-wide study in breast cancer and described single-nucleotide polymorphism located in ARHGAP10 (15). Luo et al identified a decreased ARHGAP10 expression within ovarian cancer and suggested that ARHGAP10 may serve as a biomarker for the prognosis of ovarian cancer (16). The present study demonstrated that ARHGAP10 expression was decreased within the tumor tissue of patients with lung cancer as compared to normal lung tissues from the same patients. These results were confirmed by the analysis on the TCGA dataset. Our study suggests that ARHGAP10 may serve as a useful biomarker of lung cancer.

The effects of ARHGAP10 on the biological behaviors of lung cancer were examined by increasing its expression in the two cell lines. We found that ARHGAP10 overexpression significantly suppressed cell proliferation, and inhibited cell invasion and migration. Similar functions of ARHGAP10 have been observed in ovarian cancer cells (16). Our data and this previous study demonstrated the tumor suppressor role of ARHGAP10 in various types of cancer.

GSEA, a powerful computer-based program was applied to identify the exact pathways that ARHGAP10 may regulate in lung cancer. We found that metastasis and Wnt signaling pathways were negatively associated with ARHGAP10 expression.

Cell metastasis and invasion are two main characteristics of cancer. MMP-2 and MMP-9 belong to MMPs, which degrade the extracellular matrix components and are essential for tumor metastasis by promoting cell invasion (21). VEGF is a key target of tumor cell invasion and metastasis (22). Increased expression of MMP-2, MMP-9 and VEGF is frequently observed in the most aggressive tumors $(23,24)$. ARHGAP10 overexpression significantly decreased the expression of VEGF, MMP-9 and MMP-2, which may explain its inhibitory effects on lung cancer cell invasion and migration.

The Wnt pathway plays diverse roles in the progression, metastasis and initiation of various types of cancer (25). c-Myc, a target gene of Wnt/ $\beta$-catenin signaling suppresses the accumulation of p21, a cyclin-dependent kinase (CDK) inhibitor (26). In the present study, ARHGAP10 overexpression 
caused a significant decrease in $\beta$-catenin and c-Myc, and an arresting reduction of $\mathrm{p} 21$. Moreover, the stimulation effects of $\mathrm{LiCl}$, a GSK3 $\beta$ inhibitor, on the accumulation of $\beta$-catenin were notably suppressed by ARHGAP10 overexpression. Our data suggest that ARHGAP10 has a critical role in regulating the $\mathrm{Wnt} / \beta$-catenin pathway.

To conclude, our study shows that ARHGAP10 is downregulated in lung cancer specimens. Ectopic expression of ARHGAP10 suppresses the migration, invasion and proliferation produced by lung cancer cells. ARHGAP10 affects the metastasis and Wnt signaling pathways in lung cancer. ARHGAP10 may therefore be a novel target for the treatment of lung cancer.

\section{References}

1. Jemal A, Bray F, Center MM, Ferlay J, Ward E and Forman D: Global cancer statistics. CA Cancer J Clin 61: 69-90, 2011.

2. Wang T, Nelson RA, Bogardus A and Grannis FW Jr: Five-year lung cancer survival: Which advanced stage nonsmall cell lung cancer patients attain long-term survival? Cancer 116 : $1518-1525,2010$

3. Pao W and Girard N: New driver mutations in non-small-cell lung cancer. Lancet Oncol 12: 175-180, 2011.

4. Couraud S, Zalcman G, Milleron B, Morin F and Souquet PJ: Lung cancer in never smokers - a review. Eur J Cancer 48 : 1299-1311, 2012.

5. Cornfield J, Haenszel W, Hammond EC, Lilienfeld AM, Shimkin MB and Wynder EL: Smoking and lung cancer: Recent evidence and a discussion of some questions. 1959. Int J Epidemiol 38: 1175-1191, 2009.

6. Ding L, Getz G, Wheeler DA, Mardis ER, McLellan MD, Cibulskis K, Sougnez C, Greulich H, Muzny DM, Morgan MB, et al: Somatic mutations affect key pathways in lung adenocarcinoma. Nature 455: 1069-1075, 2008.

7. Shtivelman E, Hensing T, Simon GR, Dennis PA, Otterson GA, Bueno R and Salgia R: Molecular pathways and therapeutic targets in lung cancer. Oncotarget 5: 1392-1433, 2014.

8. Bassères DS, Tizzei EV, Duarte AA, Costa FF and Saad ST: ARHGAP10, a novel human gene coding for a potentially cytoskeletal Rho-GTPase activating protein. Biochem Biophys Res Commun 294: 579-585, 2002.

9. Dubois T, Paléotti O, Mironov AA, Fraisier V, Stradal TE, De Matteis MA, Franco M and Chavrier P: Golgi-localized GAP for $\mathrm{Cdc} 42$ functions downstream of ARF1 to control Arp2/3 complex and F-actin dynamics. Nat Cell Biol 7: 353-364, 2005.

10. Lazarini M, Traina F, Machado-Neto JA, Barcellos KS, Moreira YB, Brandão MM, Verjovski-Almeida S, Ridley AJ and Saad ST: ARHGAP21 is a RhoGAP for RhoA and RhoC with a role in proliferation and migration of prostate adenocarcinoma cells. Biochim Biophys Acta 1832: 365-374, 2013.

11. Sousa S, Cabanes D, Archambaud C, Colland F, Lemichez E, Popoff M, Boisson-Dupuis S, Gouin E, Lecuit M, Legrain P, et al: ARHGAP10 is necessary for alpha-catenin recruitment at adherens junctions and for Listeria invasion. Nat Cell Biol 7: 954-960, 2005.
12. Borges L, Bigarella CL, Baratti MO, Crosara-Alberto DP, Joazeiro PP, Franchini KG, Costa FF and Saad ST: ARHGAP21 associates with FAK and PKCzeta and is redistributed after cardiac pressure overload. Biochem Biophys Res Commun 374: 641-646, 2008

13. Anthony DF, Sin YY, Vadrevu S, Advant N, Day JP, Byrne AM, Lynch MJ, Milligan G, Houslay MD and Baillie GS: $\beta$-Arrestin 1 inhibits the GTPase-activating protein function of ARHGAP21, promoting activation of RhoA following angiotensin II type 1A receptor stimulation. Mol Cell Biol 31: 1066-1075, 2011.

14. Wong NC, Bhadri VA, Maksimovic J, Parkinson-Bates M, Ng J, Craig JM, Saffery R and Lock RB: Stability of gene expression and epigenetic profiles highlights the utility of patient-derived paediatric acute lymphoblastic leukaemia xenografts for investigating molecular mechanisms of drug resistance. BMC Genomics 15: 416, 2014.

15. Azzato EM, Pharoah PD, Harrington P, Easton DF, Greenberg D, Caporaso NE, Chanock SJ, Hoover RN, Thomas G, Hunter DJ, et al: A genome-wide association study of prognosis in breast cancer. Cancer Epidemiol Biomarkers Prev 19: 1140-1143, 2010.

16. Luo N, Guo J, Chen L, Yang W, Qu X and Cheng Z: ARHGAP10, downregulated in ovarian cancer, suppresses tumorigenicity of ovarian cancer cells. Cell Death Dis 7: e2157, 2016.

17. Subramanian A, Tamayo P, Mootha VK, Mukherjee S, Ebert BL, Gillette MA, Paulovich A, Pomeroy SL, Golub TR, Lander ES, et al: Gene set enrichment analysis: A knowledge-based approach for interpreting genome-wide expression profiles. Proc Natl Acad Sci USA 102: 15545-15550, 2005.

18. Teng Y, Wang X, Wang Y and Ma D: Wnt $/ \beta$-catenin signaling regulates cancer stem cells in lung cancer A549 cells. Biochem Biophys Res Commun 392: 373-379, 2010.

19. Bigarella CL, Borges L, Costa FF and Saad ST: ARHGAP21 modulates FAK activity and impairs glioblastoma cell migration. Biochim Biophys Acta 1793: 806-816, 2009.

20. Carles A, Millon R, Cromer A, Ganguli G, Lemaire F, Young J, Wasylyk C, Muller D, Schultz I, Rabouel Y, et al: Head and neck squamous cell carcinoma transcriptome analysis by comprehensive validated differential display. Oncogene 25: 1821-1831, 2006.

21. Bogenrieder T and Herlyn M: Axis of evil: Molecular mechanisms of cancer metastasis. Oncogene 22: 6524-6536, 2003.

22. Su JL, Yang PC, Shih JY, Yang CY, Wei LH, Hsieh CY, Chou CH, Jeng YM, Wang MY, Chang KJ, et al: The VEGF-C/Flt-4 axis promotes invasion and metastasis of cancer cells. Cancer Cell 9: 209-223, 2006.

23. Cockett MI, Murphy G, Birch ML, O'Connell JP, Crabbe T, Millican AT, Hart IR and Docherty AJ: Matrix metalloproteinases and metastatic cancer. Biochem Soc Symp 63: 295-313, 1998

24. Kerbel RS and Kamen BA: The anti-angiogenic basis of metronomic chemotherapy. Nat Rev Cancer 4: 423-436, 2004.

25. Polakis P: Wnt signaling in cancer. Cold Spring Harb Perspect Biol 4: a008052, 2012. doi:10.1101/cshperspect.a008052.

26. Tang Y, Simoneau AR, Liao WX, Yi G, Hope C, Liu F, Li S, Xie J, Holcombe RF, Jurnak FA, et al: WIF1, a Wnt pathway inhibitor, regulates SKP2 and c-myc expression leading to G1 arrest and growth inhibition of human invasive urinary bladder cancer cells. Mol Cancer Ther 8: 458-468, 2009. 